\title{
A Semi-Heuristic Process-Reaction Curve PID Controller Tuning Method
}

\author{
Christer Dalen, David Di Ruscio \\ University College of Southeast Norway, P.O. Box 203, N-3901 Porsgrunn, Norway. E-mail: \\ David.Di.Ruscio@usn.no
}

\begin{abstract}
A simple semi-heuristic method for designing PID controllers for time constant models are shown to be close to optimal. A Process-Reaction Curve method is proposed, composed by a method for approximating stable time constant models with an unstable DIPTD model, and relative time delay error $\delta$-PID controller tuning. The Pareto-Optimal controller is used as reference.
\end{abstract}

Keywords: PID controller, tuning, double integrating system, relative time delay error, semi-heuristic, robustness, performance, Pareto-Optimal, Process-Reaction Curve, Ziegler-Nichols, lag

\section{Introduction}

By heuristic, we mean that the method has evolved based on practical implementations or simulations. The most known examples of heuristic PID controller $\left(h_{c}(s)=K_{p}\left(1+\frac{1}{T_{i} s}+T_{d} s\right)\right)$ tuning methods are given in Ziegler and Nichols (1942) (ZN), which proposed two methods, a Process-Reaction Curve (PRC) method and an ultimate gain method, i.e. based on open- (reaction curve) and closed loop (with $\mathrm{P}$ controller) transient step responses, i.o.

In this paper, PRC methods are at focus, where the ZN method has the following tuning rule for a PID controller,

$$
\begin{aligned}
K_{p} & =\frac{1.2}{R L} \\
T_{i} & =2 L \\
T_{d} & =\frac{L}{2}
\end{aligned}
$$

where

$$
\begin{aligned}
R & =\max _{t} \frac{d y}{d t} \\
L & =t_{1}-\frac{y_{1}}{R}
\end{aligned}
$$

are the maximum slope of curve (reaction rate) and the lag, i.o., and, $t_{1}=\arg R, y_{1}=y\left(t_{1}\right)$. This is illustrated in Figure 1. See App. 4 for proof of Eq. (5).

In this work, a semi-heuristic PRC PID controller tuning method is proposed, i.e. a simple, $K$, and, $\tau$, estimation rule for a Double Integrator Plus Time Delay (DIPTD) model $\left(h_{p}(s)=K \frac{1}{s^{2}} e^{-\tau s}\right)$, based on a reaction curve for a stable (possible) higher order system or model, is proposed. Thereafter, the newly published $\delta$-PID controller tuning method, Di Ruscio and Dalen (2017) is used, where a relative time delay error, $\delta>0$, is prescribed. Note, that other PRC methods as ZN and Cohen-Coon (Cohen and Coon (1952)) are fixed, i.e. there are no tuning parameters. Hence, these methods may perform poorly in some situations.

Notice that, noise is not considered, i.e. only deterministic model or system responses are given. Cases with noise is recommended to be dealt with using proper system identification methods, e.g., Ljung (1999), Di Ruscio (1996) and Di Ruscio (2009), thereafter, possible DIPTD model approximations may be done.

Only reaction curves of following process models are assumed in this work,

$$
h_{p}(s)=\frac{k}{\prod_{j=1}^{n}\left(T_{j} s+1\right)},
$$




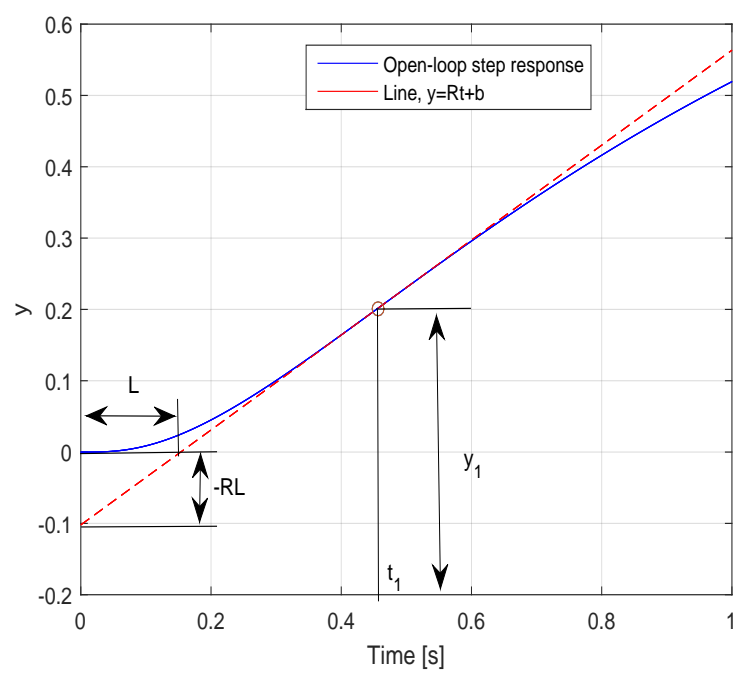

Figure 1: Lag, $L$, and reaction rate, $R$, illustrated on the reaction curve from the process model as given in row 1 , column 1 in Table 1 .

where, $T_{1} \geq T_{2} \geq \cdots \geq T_{n} \geq 0$, and, $n>1$.

The contributions in this paper may be itemized as follows.

- It is proposed to approximate a stable time constant model as in Eq. (6) with an unstable DIPTD model.

- A semi-heuristic method for tuning PID controller tuning based on reaction curve is proposed.

- The proposed method is compared vs. the SIMC method in numerous examples where ParetoOptimal controllers are used as references.

All numerical calculations and plotting facilities are provided by using the MATLAB software, MATLAB (2016). The rest of this paper is organized as follows. In Sec. 2 the semi-heuristic method is presented. In Sec. 3 numerical results are shown. Lastly, discussion and concluding remarks are given in Sec. 4.

\section{Theory}

\subsection{Semi-Heuristic PID Controller Tuning}

The following semi-heuristic DIPTD estimation rules based on the reaction curve is proposed,

$$
\begin{aligned}
K & =\frac{R}{L}, \\
\tau & =\frac{L}{2 \pi},
\end{aligned}
$$

where the reaction rate, $R$, and the lag, $L$, are given by Eqs. (4) and (5), i.o. See App. B for proof of the gain acceleration, $K$, in Eq. (7). Note that, the rule for the time delay, $\tau$, in Eq. (8), is chosen heuristic.

\section{Algorithm 2.1 (Semi-Heuristic PID Tuning)}

1. Find the lag, $L$, and the reaction rate, $R$, using Eqs. (4) and (5), based on the reaction curve.

2. Find the gain acceleration, $K$, and time delay, $\tau$, in the DIPTD model, using Eqs. (7) and (8).

3. Obtain the PID controller parameters, $K_{p}, T_{d}$, and, $T_{i}$, using $\delta$-PID controller tuning, i.e. Alg. 2.1 and Eq. (27) in Di Ruscio and Dalen (2017), i.o.

The presented Alg. 2.1 is used to tune PID controllers for a wide range of examples in Sec. 3.

\subsection{ZN and Alg. 2.1 PID Connection}

The $\delta$-PID controller tuning parameters (Alg. 2.1 and Eq. (27) in Di Ruscio and Dalen (2017)) can be described as,

$$
\begin{aligned}
K_{p}^{*} & =f_{1}(c, \delta, K, \tau), \\
T_{i}^{*} & =\gamma T_{d}^{*}, \\
T_{d}^{*} & =f_{2}(c, \delta, \tau),
\end{aligned}
$$

where the relative time delay error, $\delta$, is the tuning parameter, $c$, and, $\gamma$, are, in general, user-chosen options.

We want to solve the following set of equations wrt., $\delta$

$$
\begin{aligned}
K_{p}^{*}(c, \delta, R, L) & =K_{p}(L, R), \\
T_{i}^{*}\left(c, \delta_{2}, \gamma, L\right) & =T_{i}(L), \\
T_{d}^{*}(c, \delta, L) & =T_{d}(L),
\end{aligned}
$$

where, $K_{p}^{*}$, and, $T_{d}^{*}$, are the PID controller parameters from Alg. 2.1, and, $K_{p}$, and, $T_{d}$, are the ZN PID controller tuning parameters in Eqs. (1)-(3).

By setting the solutions for, $\delta$, from Eqs. (12) and (14) equal, we must have that

$$
\begin{aligned}
\delta & = \\
\pm \sqrt{\frac{10}{3} \pi \frac{\operatorname{atan}(c \sqrt{f})}{\sqrt{c f}}-1} & =-\frac{c \sqrt{f}-\pi \operatorname{atan}(c \sqrt{f})}{c \sqrt{f}} \\
c & =\frac{3}{10},
\end{aligned}
$$

where, $f$, is a function of c, defined by Eq. (11) in Di Ruscio and Dalen (2017). Comparing Eqs. (2) with (3) it is needed that, $\gamma=4$.

A consequence now is that the $\mathrm{ZN}$ and Alg. 2.1 PID controllers are equal. 


\subsection{Equivalent ZN DIPTD tuning}

Consider the ZN tuning rules for PI control,

$$
\begin{aligned}
K_{p}^{*} & =\frac{0.9}{R L} \\
T_{i} & =\frac{L}{0.3}
\end{aligned}
$$

The ZN PRC method for PI tuning for IPTD (Integrator Plus Time Delay) processes $\left(h_{p}(s)=K \frac{e^{-\tau s}}{s}\right)$, with step response is equivalent to PD control for DIPTD process with impulse response. PD tuning rules may be deduced for a DIPTD plant, simply by replacing, $K_{p}^{*}$ and $T_{i}$ in the PI tuning algorithm, in Eq. (18) and (19), for the IPTD system with, $K_{p} T_{d}$, and, $T_{d}$, i.o. (p. 96, Di Ruscio and Dalen (2017)), viz.

$$
\begin{aligned}
T_{d} & :=T_{i}=\frac{L}{0.3} \\
K_{p} & :=\frac{K_{p}^{*}}{T_{d}}=\frac{0.27}{R L^{2}} .
\end{aligned}
$$

These results are not considered further.

\section{Numerical Results}

In the incoming, Alg. 2.1 is compared vs. the modelbased SIMC method. The SIMC method is defined as (possible) model reduction (half rule) to second order model, i.e. Eqs. (11) in Skogestad (2003), then PID controller tuning given by row 2 in Table 1 in Skogestad (2003) (row 5 for a type of integrating process in Example 9), is used. Note that, these parameters correspond to a PID controller on cascade form, hence we need to convert them to ideal form.

For step 3 in Alg. 2.1, i.e. $\delta$-PID controller tuning, $\gamma=c=2.12$, is used, which was justified in an earlier paper Dalen and Di Ruscio (2017). Note that, it could have been possible to compare against the optimization method proposed in Dalen and Di Ruscio (2017), however in this paper, only simple rule-based methods are of interest.

For comparing the performances of the PID controllers consider the following mean square error criterion

$$
\Gamma_{m}=\frac{1}{M}\left(J_{P O}-J_{m}\right)^{T}\left(J_{P O}-J_{m}\right),
$$

where, $J_{m} \in \mathbb{R}^{M}$, is the performance generated from the given tuning method, $m, J_{P O} \in \mathbb{R}^{M}$, is the ParetoOptimal (PO) performance generated using the gradient optimization method (Grimholt and Skogestad (2016)), and, $\mathrm{M}=\operatorname{length}\left(J_{m}\right)$. The reader is encouraged to see Grimholt and Skogestad (2016) and previous papers Skogestad and Grimholt (2012), Dalen and
Di Ruscio (2017) and Di Ruscio and Dalen (2017) for details about the optimization problem.

In the following, we take a shot at covering a good amount of typical industrial processes, see Table 1. The trade-off curves are illustrated for Example 1, 7 and 11 (process model in row 1, 7, 11 in column 2 in Table 1) in Figures 2, 3 and 4. In Figure 5 the effect, in terms of magnitude responses, of varying the gain acceleration, $K$, in the DIPTD model, is illustrated. The DIPTD approximations and performance measures, $\Gamma_{m}$, are shown in Table 2. Comparison of the controllers from Alg. 2.1 and PO are presented in Table 3 (for prescribed robustness, $M_{s}=1.59$ ), and the corresponding margins are given in Tables 4 and 5 (App. C for PO).



Figure 2: Example 1: Performance vs. robustness, $J$ vs. $M_{s}$ trade-off curves. Comparing Alg. 2.1, SIMC vs. the PO-PID controllers for the process model at row 1, column 2 in Table 1 . The method performance measures, i.e. $\Gamma_{m}$ in Eq. (22), are given in Table 2. The tuning parameter in the SIMC method, $T_{c}$, is the time constant for the set-point response 


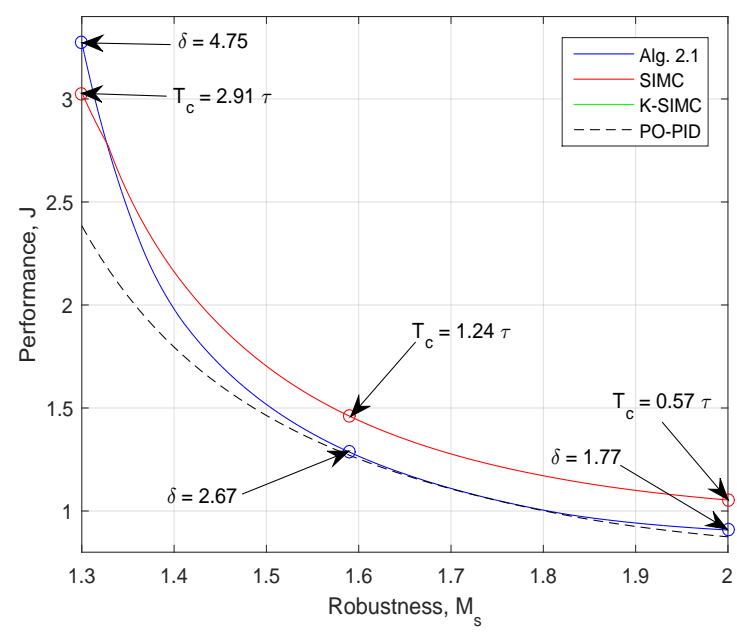

Figure 3: Example 7: Performance vs. robustness, $J$ vs. $M_{s}$ trade-off curves. Comparing Alg. 2.1, SIMC vs. the PO-PID controllers for the process model at row 7 , column 2 in Table 1 .

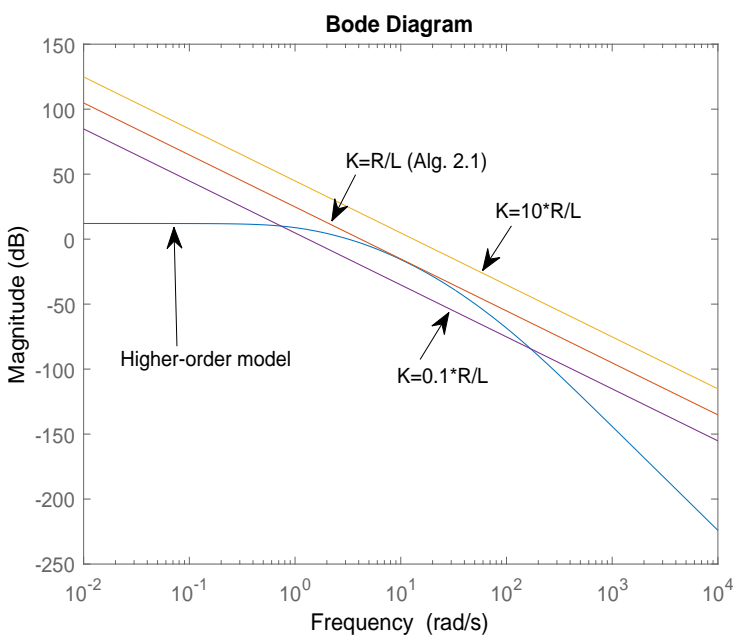

Figure 5: Example 1: Shows the magnitude responses for the higher order process and the DIPTD model. Illustrates the effect of varying the gain acceleration, $K$, in the DIPTD model.

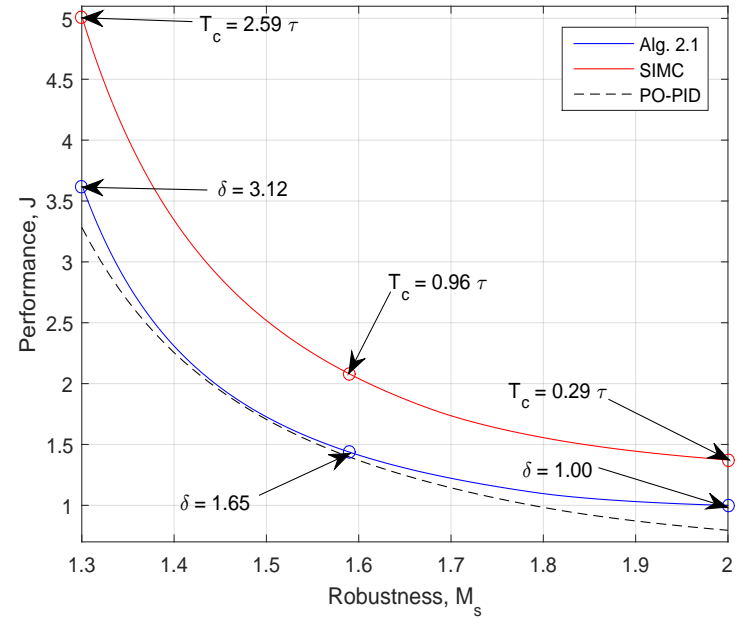

Figure 4: Example 11: Performance vs. robustness, $J$ vs. $M_{s}$ trade-off curves. Comparing Alg. 2.1, SIMC vs. the PO-PID controllers for the process model at row 11, column 2 in Table 1. The method performance measures, i.e. $\Gamma_{m}$ in Eq. (22), are given in Table 2.
Table 1: Shows the test batch, i.e. process model examples used in the numerical simulations. $\dagger$ means that the gain, $k$, was not given, but, $k=1$, is assumed.

\begin{tabular}{|c|c|c|}
\hline $\mathrm{E}$ & Process model & Reference \\
\hline  & $\begin{array}{c}\text { Process incl. sensor }+ \text { valve } \\
\frac{1}{(s+1)(0.2 s+1)(0.04 s+1)(0.008 s+1)}\end{array}$ & $\begin{array}{l}\text { Seborg et al. (2004) } \\
\text { Aström et al. (1998) }\end{array}$ \\
\hline 2 & $\begin{array}{l}\text { Bioseperation process } \\
\frac{2}{(5 s+1)(3 s+1)(s+1)}\end{array}$ & $\begin{array}{l}\text { p. } 183 \\
\text { Seborg et al. (2004) }\end{array}$ \\
\hline 3 & $\begin{array}{l}\text { Perfectly stirred tank } \\
\frac{10}{(5 s+1)(s+1)(0.2 s+1)}\end{array}$ & $\begin{array}{l}\text { p. } 334 \\
\text { Seborg et al. (2004) }\end{array}$ \\
\hline 4 & $\begin{array}{l}\text { Cascaded vessels } \\
\frac{k}{(40 s+1)(40 s+1)(10 s+1)} \dagger\end{array}$ & Balchen and Lie (1987) \\
\hline 5 & $\frac{0.1667}{(s+1)(0.5 s+1)(0.3333 s+3)}$ & Coughanowr (1991) \\
\hline 6 & $\frac{1}{(s+1)^{3}}$ & $\begin{array}{l}\text { Balchen (1958) } \\
\text { Aström et al. (1998) }\end{array}$ \\
\hline 7 & $\frac{5}{(10 s+1)(4 s+1)(s+1)(0.2 s+1)}$ & Seborg et al. (2004) \\
\hline 8 & $\frac{12}{(8 s+1)(2 s+1)(0.4 s+1)(0.1 s+1)}$ & Seborg et al. (2004) \\
\hline 9 & $\frac{250}{s(0.05 s+1)(0.005 s+1)}$ & Balchen et al. (1999) \\
\hline 10 & $\frac{6}{(6 s+1)(4 s+1)(2 s+1))}$ & R. Kumar (2015) \\
\hline 11 & $\begin{array}{l}\text { Distillation column } \\
\frac{34}{(54 s+1)(0.5 s+1)^{2}}\end{array}$ & $\begin{array}{l}\text { p. } 591 \\
\text { Luyben (1990) }\end{array}$ \\
\hline 12 & $\begin{array}{l}\text { Heat exchanger } \\
\frac{0.8}{(30 s+1)(10 s+1)(3 s+1)}\end{array}$ & $\begin{array}{l}\text { p. } 315 \\
\text { Smith and Corripio (1997) }\end{array}$ \\
\hline
\end{tabular}


Table 2: Comparing Alg. 2.1 vs. the SIMC controllers. Column 2 shows the DIPTD model from the heuristic rule, i.e. step 2 in Alg. 2.1. Column 3 shows the performance, $\Gamma_{m}$, given by Eq. (22). The column 4 tells how many times better Alg. 2.1 is than the SIMC.

\begin{tabular}{|l|l|l|l|}
\hline $\mathrm{E}$ & Approximation & $\Gamma_{\text {Alg } 2.1}$ & $\frac{\Gamma_{\text {SIMC }}}{\Gamma_{\text {Alg } 2.1}}$ \\
\hline \hline 1 & $4.3378,0.0244$ & 0.0072 & 8.7 \\
\hline 2 & $0.0928,0.3056$ & 0.0217 & 8.8 \\
\hline 3 & $1.8302,0.1158$ & 0.0507 & 4.3 \\
\hline 4 & $5 \mathrm{E}-04,3.1512$ & 0.0212 & 9.0 \\
\hline 5 & $0.1715,0.0687$ & 0.0460 & 4.7 \\
\hline 6 & $0.3360,0.1282$ & 0.1170 & 4.9 \\
\hline 7 & $0.0953,0.4462$ & 0.0430 & 1.8 \\
\hline 8 & $0.6350,0.2353$ & 0.0174 & 4.2 \\
\hline 9 & $4 \mathrm{E}+03,0.0088$ & 0.1054 & 3.5 \\
\hline 10 & $0.1411,0.4565$ & 0.0457 & 4.4 \\
\hline 11 & $0.6466,0.1469$ & 0.0152 & 45 \\
\hline 12 & $0.0021,1.1520$ & 0.0227 & 8.9 \\
\hline
\end{tabular}

Table 3: Comparing Alg. 2.1 vs. the PO controllers for prescribed robustness, $M_{s}=1.59$ (SIMCtuned PI, $h_{p}(s)=\frac{1}{s+1} e^{-s}$ Skogestad and Grimholt (2012)). Columns 2 and 3 show the PID controller parameters, $K_{p}, T_{i}$, and, $T_{d}$, for the given method and ex. in Table 1.

\begin{tabular}{|l|l|l|}
\hline $\mathrm{E}$ & $\mathrm{PO}$ & Alg. 2.1 \\
\hline \hline 1 & $24.5,0.30,0.14$ & $24.6,0.30,0.14$ \\
\hline 2 & $5.56,3.41,2.52$ & $5.52,4.31,2.03$ \\
\hline 3 & $4.27,1.00,0.72$ & $4.03,1.14,0.54$ \\
\hline 4 & $11.0,35.1,26.1$ & $10.9,44.2,20.9$ \\
\hline 5 & $47.4,0.77,0.60$ & $47.5,1.08,0.51$ \\
\hline 6 & $6.09,1.35,1.14$ & $6.10,2.15,1.02$ \\
\hline 7 & $2.15,6.16,2.95$ & $2.10,6.90,3.26$ \\
\hline 8 & $1.54,3.02,1.40$ & $1.52,3.14,1.48$ \\
\hline 9 & $1.63,0.03,0.02$ & $1.50,0.04,0.02$ \\
\hline 10 & $1.34,5.12,3.96$ & $1.34,7.10,3.35$ \\
\hline 11 & $5.51,1.35,0.92$ & $5.48,1.64,0.77$ \\
\hline 12 & $22.5,12.0,8.65$ & $22.0,14.4,6.77$ \\
\hline
\end{tabular}

Table 4: The columns 2:6 show the Phase Margin (PM), Gain Margin (GM), relative time delay error $\delta$, maximum time delay error, $d \tau_{\max }=$ $\delta \tau$, and the Delay Margin (DM), for Alg. 2.1. $M_{s}=1.59$.

\begin{tabular}{|l|l|l|l|l|l|}
\hline $\mathrm{E}$ & $\mathrm{PM}$ & $\mathrm{GM}$ & $\delta$ & $\delta \tau$ & $\mathrm{DM}$ \\
\hline \hline 1 & 47.8 & 8.2 & 1.9 & 0.05 & 0.06 \\
\hline 2 & 45.9 & $\infty$ & 2.3 & 0.72 & 0.80 \\
\hline 3 & 43.5 & $\infty$ & 2.2 & 0.15 & 0.22 \\
\hline 4 & 45.6 & $\infty$ & 2.3 & 7.34 & 8.20 \\
\hline 5 & 47.1 & $\infty$ & 2.7 & 0.19 & 0.20 \\
\hline 6 & 47.6 & $\infty$ & 3.0 & 0.38 & 0.38 \\
\hline 7 & 51.0 & 7.4 & 2.7 & 1.19 & 1.35 \\
\hline 8 & 49.3 & 7.2 & 2.2 & 0.51 & 0.62 \\
\hline 9 & 40.8 & $\infty$ & $8 \mathrm{E}-3$ & $7 \mathrm{E}-5$ & $6.2 \mathrm{E}-3$ \\
\hline 10 & 46.8 & $\infty$ & 2.7 & 1.23 & 1.29 \\
\hline 11 & 46.1 & $\infty$ & 1.6 & 0.24 & 0.31 \\
\hline 12 & 44.7 & $\infty$ & 2.0 & 2.26 & 2.72 \\
\hline
\end{tabular}

\section{Discussion and Concluding Remarks}

The discussion and concluding remarks are itemized as follows.

- Approximating stable responses with unstable (DIPTD) processes may be seen as a provoking idea, however quite surprising, this semi-heuristic method can be argued to be close to optimal in numerous examples. See Table 2.

- In this paper, it is seen that Alg. 2.1 has an edge over SIMC, viz. in rows 1:12 in column 4, in Table 2, Alg. 2.1 is at minimum, $\frac{\Gamma_{S I M C}}{\Gamma_{A l g .2 .1}}=1.8$, and at maximum, $\frac{\Gamma_{S I M C}}{\Gamma_{\text {Alg.2.1 }}}=45$, times better than SIMC. Notice, that by using the ideal instead of the cascade PID controller form may have advantages in terms of optimality.

- Notice, that the results in Tables 2-4 are based on the original models given in Table 1 . The approximated models are only used for controller design.

- Note that, it is not recommended using Alg. 2.1 for processes which have multiple poles (or close), with exception of, $n=2,3$, which through additional simulations have given acceptable results.

- Notice, that the tuning parameter, $\delta=2.3$, i.e. mean of column 4 (row 1:12) in Table 4 , seems like a reasonable initial tuning parameter choice. The exception is the integrating process in Example 9.

- In columns 5 and 6 in Table 4 , it is seen that the prescribed maximum time delay error, $d \tau_{\max }=$ $\delta \tau$, is close and less than the Delay Margin.

- Notice that, step. 2 in Alg. 2.1 is, in general, easier to use than the half rule reduction technique, which is the 1st step in the SIMC method (Skogestad (2003)).

- Note that, the optimization based method in Dalen and Di Ruscio (2017) is not necessary $\Gamma$ optimal (minimizing Eq. (22)), hence, the proposed PRC method may in some cases outperform the optimization method. However, note that in this paper, only process models as in Eq. (6) are assumed, and in Dalen and Di Ruscio (2017) also nonlinear models were considered.

- Remarkably, this simple heuristic PRC PID tuning Alg. 2.1 based on the DIPTD model approximation Eqs. (7) and (8) gives PID tuning rules which are close to optimal (PO), i.e. approximately minimizing the objective Eq. (22). 


\section{A. Proof of Eq. (4)}

The line, $y=R t+b$, and, $R$, are known.

Consider the line, $y=R t+b$, by inserting, $t=L$, and, $y=0$, gives, $0=R L+b \Rightarrow b=-R L$.

Hence, $y=R t-R L$, or, $y_{1}=R t_{1}-R L \Rightarrow L=t_{1}-\frac{y_{1}}{R}$.

\section{B. Proof of Eq. (7)}

The proposed heuristic rule for estimating the gain acceleration, $K$, in Eq. (7), may be justified by considering the inverse Laplace transform of a DIPTD model $\left(h_{p}(s)=K \frac{e^{-\tau s}}{s^{2}}\right)$, and considering an unit step, $u(t-\tau)=1$, viz. it may shown that, $\ddot{y}=K u(t-\tau) \Rightarrow$ $\dot{y}=K u \int_{0}^{t_{1}} d t \Rightarrow R=K u\left(t_{1}-C\right) \Rightarrow K=\frac{R}{L}$. Where Eq. (5) and, $C=t_{1}-L=\frac{y_{1}}{R}$, are used, which is found reasonable from numerous simulations.

\section{Margins for the PO controllers}

Table 5: The columns 2:4 show the Phase Margin (PM), Gain Margin (GM) and the Delay Margin (DM), for PO-PID.

\begin{tabular}{|l|l|l|l|}
\hline E & PM & GM & DM \\
\hline 1 & 48.0 & 8.2 & 0.06 \\
\hline 2 & 46.7 & $\infty$ & 0.72 \\
\hline 3 & 45.2 & $\infty$ & 0.18 \\
\hline 4 & 46.6 & $\infty$ & 7.31 \\
\hline 5 & 47.5 & $\infty$ & 0.18 \\
\hline 6 & 47.8 & $\infty$ & 0.37 \\
\hline 7 & 49.6 & 7.7 & 1.39 \\
\hline 8 & 48.3 & 7.4 & 0.63 \\
\hline 9 & 44.1 & $\infty$ & $5 \mathrm{E}-3$ \\
\hline 10 & 47.3 & $\infty$ & 1.20 \\
\hline 11 & 46.9 & $\infty$ & 0.28 \\
\hline 12 & 46.1 & $\infty$ & 2.37 \\
\hline
\end{tabular}

\section{References}

Åström, K. J., Panagopoulos, H., and Hägglund, T. Design of pi controllers based on non-convex optimization. Automatica, 1998. 34(5):585-601. doi:10.1016/S0005-1098(98)00011-9.

Balchen, J. A Performance Index for Feedback Control Systems Based on the Fourier Transform of the Control Deviation. Acta polytechnica Scandinavica: Mathematics and computing machinery series. Norges tekniske vitenskapsakademi, 1958.

Balchen, J. G., Andresen, T., and Foss, B. A. Reguleringsteknikk. Tapir, 1999. In norwegian. First edition.
Balchen, J. G. and Lie, B. An Adaptive Controller Based upon Continuous Estimation of the Closed Loop Frequency Response. Modeling, Identification and Control, 1987. 8(4):223-240. doi:10.4173/mic.1987.4.3.

Cohen, G. and Coon, G. Theoretical consideration of retarded control. Transactions of ASME, 1952. 75(1):827-834. doi:10.4173/mic.2017.4.3.

Coughanowr, D. Process Systems Analysis and Control. McGraw-Hill chemical engineering series. McGraw-Hill, 1991.

Dalen, C. and Di Ruscio, D. PD/PID controller tuning based on model approximations: Model reduction of some unstable and higher order nonlinear models. Modeling, Identification and Control, 2017. 38(4):185-197. doi:10.4173/mic.2017.4.3.

Di Ruscio, D. Combined Deterministic and Stochastic System Identification and Realization: DSR - A Subspace Approach Based on Observations. Modeling, Identification and Control, 1996. 17(3):193-230. doi:10.4173/mic.1996.3.3.

Di Ruscio, D. Closed and Open Loop Subspace System Identification of the Kalman Filter. Modeling, Identification and Control, 2009. 30(2):71-86. doi:10.4173/mic.2009.2.3.

Di Ruscio, D. and Dalen, C. Tuning PD and PID Controllers for Double Integrating Plus Time Delay Systems. Modeling, Identification and Control, 2017. 38(2):95-110. doi:10.4173/mic.2017.2.4.

Grimholt, C. and Skogestad, S. Optimization of fixedorder controllers using exact gradients. 2016. URL http://folk.ntnu.no/skoge/publications/ 2016/grimholt-jpc-pid-exact-gradient/main . pdf. Unpublished.

Ljung, L. System Identification (2nd ed.): Theory for the User. Prentice Hall PTR, Upper Saddle River, NJ, USA, 1999.

Luyben, W. Process Modeling, Simulation, and Control for Chemical Engineers. Chemical engineering series. McGraw-Hill, 1990.

MATLAB. Version 9.1.0.441655 (R2016b). The MathWorks Inc., Natick, Massachusetts, USA, 2016. Control System Toolbox, Version 9.3. Optimization Toolbox, Version 6.2.

R. Kumar, V. C., S.K. Singla. Comparison among some well known control schemes with different tuning methods. Journal of Applied Research and Technology, 2015. 13(3):409 - 415. doi:10.1016/j.jart.2015.07.007. 
Seborg, D., Edgar, T., and Mellichamp, D. Process dynamics and control. Wiley series in chemical engineering. Wiley, 2004.

Skogestad, S. Simple analytic rules for model reduction and PID controller tuning. Journal of Process Control, 2003. 13(13):291-309. doi:10.1016/S09591524(02)00062-8.

Skogestad, S. and Grimholt, C. The SIMC Method for Smooth PID Controller Tuning, pages 147-175. Springer London, London, 2012. doi:10.1007/978-14471-2425-2_5.

Smith, C. and Corripio, A. Principles and practice of automatic process control. J. Wiley, 1997.

Ziegler, J. and Nichols, N. B. Optimum settings for automatic controllers. Trans. of the A.S.M.E., 1942. 64(64):759-768. 